\title{
Consensus recommendation for a diagnostic guideline for acid sphingomyelinase deficiency
}

\author{
Margaret M. McGovern, MD, PhD1', Carlo Dionisi-Vici, MD², Roberto Giugliani, MD, PhD, \\ Paul Hwu, MD, PhD ${ }^{4}$, Olivier Lidove, MD, Zoltan Lukacs, $\mathrm{PhD}^{6}$, Karl Eugen Mengel, $\mathrm{MD}^{7}$, \\ Pramod K. Mistry, MD, PhD ${ }^{8}$, Edward H. Schuchman, $\mathrm{PhD}^{9}$ and Melissa P. Wasserstein, MD ${ }^{10}$
}

\begin{abstract}
Background: Acid sphingomyelinase deficiency (ASMD) is a rare, progressive, and often fatal lysosomal storage disease. The underlying metabolic defect is deficiency of the enzyme acid sphingomyelinase that results in progressive accumulation of sphingomyelin in target tissues. ASMD manifests as a spectrum of severity ranging from rapidly progressive severe neurovisceral disease that is uniformly fatal to more slowly progressive chronic neurovisceral and chronic visceral forms. Disease management is aimed at symptom control and regular assessments for multisystem involvement.
\end{abstract}

Purpose and methods: An international panel of experts in the clinical and laboratory evaluation, diagnosis, treatment/management, and genetic aspects of ASMD convened to review the evidence base and share personal experience in order to develop a guideline for diagnosis of the various ASMD phenotypes.

Conclusions: Although care of ASMD patients is typically provided by metabolic disease specialists, the guideline is directed at a wide range of providers because it is important for primary care providers (e.g., pediatricians and internists) and specialists (e.g., pulmonologists, hepatologists, and hematologists) to be able to identify ASMD.

Genet Med advance online publication 13 April 2017

Key Words: acid sphingomyelin deficiency; lysosomal storage disorder; Niemann-Pick disease types A and B

\section{INTRODUCTION}

Acid sphingomyelinase deficiency (ASMD), commonly known as Niemann-Pick disease (NPD) types A and B, is a rare, progressive, and often fatal lysosomal storage disorder (LSD). The metabolic defect in ASMD is deficiency of the lysosomal enzyme acid sphingomyelinase (ASM) due to mutations in the sphingomyelin phosphodiesterase 1 gene (SMPD1). ${ }^{1}$ ASM catalyzes hydrolysis of sphingomyelin to ceramide and phosphocholine, and ASM deficiency results in the progressive accumulation of sphingomyelin and other lipids within tissues that are rich in reticuloendothelial cells, including the spleen, liver, lung, bone marrow, and lymph nodes. With severe disease, neurons may also be affected. ASMD has an autosomal recessive pattern of inheritance, and the birth prevalence is estimated at $0.4-0.6 / 100,000 .^{2}$ Although NPD A is more commonly found in individuals of Ashkenazi Jewish ancestry, both forms of ASMD are pan ethnic. ASMD was first described in 1914 and is distinct from NPD C, which has a different genetic and biochemical basis as well as a distinct clinical profile from ASMD.

Currently, only supportive care is available for patients with ASMD, although enzyme replacement therapy (ERT) is in clinical development for the treatment of the nonneurologic manifestations of ASMD. ${ }^{3}$ Although the most severe infantile neurovisceral form of ASMD (NPD A) is uniformly fatal, individuals with other ASMD phenotypes may benefit from regular assessments for multisystem involvement as early as possible. However, the diagnosis of ASMD is often delayed by months to years because the complex signs and symptoms overlap with other disorders, creating a diagnostic dilemma for many practitioners.

\section{OBJECTIVES OF CONSENSUS DIAGNOSIS GUIDELINE}

The objective of this consensus document is to present an educational resource of current practices for the diagnosis of ASMD. Although care of ASMD patients is typically provided by metabolic disease specialists/medical geneticists, this guideline is directed at primary care providers (e.g., pediatricians and internist) and specialists (e.g., pulmonologists, hepatologists, and hematologists) so that they will be able to recognize these patients to provide the appropriate care and referrals.

\section{METHODS AND PROCESS}

An international group of experts in the clinical and laboratory evaluation, diagnosis, treatment/management, and genetic

\footnotetext{
${ }^{1}$ Department of Pediatrics, Stony Brook University School of Medicine, Stony Brook, New York, USA; ${ }^{2}$ Division of Metabolism, Bambino Gesù Childrens Hospital, Rome, Italy; ${ }^{3}$ Medical Genetics Service, Hospital de Clinicas de Porto Alegre, Porto Alegre, Brazil; ${ }^{4}$ Department of Medical Genetics and Pediatrics National Taiwan University Hospital, Taipei, Taiwan; ${ }^{5}$ Department of Internal Medicine - Rheumatology, Groupe Hospitalier Diaconesses-Croix St Simon, Paris, France; ${ }^{6}$ Department of Pediatrics, University Medical Center Hamburg-Eppendorf, Hamburg, Germany; ${ }^{7}$ Center for Child and Adolescent Medicine, University Medical Center of the Johannes Gutenberg University, Mainz, Germany; ${ }^{8}$ Department of Pediatrics, Yale University, New Haven, Connecticut, USA; ${ }^{9}$ Department Of Genetics and Genomic Sciences Icahn School of Medicine at Mount Sinai, New York, New York, USA; ${ }^{10}$ Department of Pediatrics Children's Hospital at Montefiore, Albert Einstein College of Medicine, Bronx, New York, USA. Correspondence: Margaret M. McGovern (Margaret.McGovern@stonybrookmedicine.edu)
} 
aspects of ASMD convened at a 1-day meeting to review the evidence base and develop a guideline for the diagnosis and management of ASMD phenotypes. Published materials as well as personal experiences were discussed. These recommendations should be considered a consensus based on expert opinion because additional levels of evidence were not always available in the literature.

\section{SUBTYPES AND NATURAL HISTORY OF ASMD}

Although all patients with ASMD share the same basic metabolic defect, a spectrum of clinical presentations and outcomes results from differences in disease severity, which contribute to the diagnostic challenges. The severity of ASMD ranges from a rapidly progressive infantile neurovisceral disease that is uniformly fatal in early childhood to more slowly progressive chronic neurovisceral and chronic visceral forms. Variability in the amount of residual enzyme activity, the extent of neurological involvement, and the rate of disease progression contribute to the phenotypic spectrum, although currently unknown modifiers are also likely to play a role. Predictions of phenotype cannot be made solely from the ASM activity determined from enzyme assays, which are usually performed white blood cells, dried blood spots (DBS), or cultured skin fibroblasts. Likely modifiers of disease severity are secondary accumulation of other lipids such as sphingosine, ceramide, or cholesterol, which are highly bioactive and can lead to cellular and other abnormalties. ${ }^{4}$ Modifier genes and/or environmental factors may also influence disease severity, although clear evidence for the impact of these variables does not currently exist.

The phenotypic variability of ASMD has led to categorization of subtypes based on severity and the degree of neurological involvement. The clinical features of the ASMD subtypes, with highlights of the similarities and differences between them, are described in Table 1. Examples of the clinical presentations and diagnostic experience for patients with different ASMD subtypes are presented in the Appendix (Case Vignettes). The clinical features and natural history of the ASMD subtypes have recently been reviewed. ${ }^{4}$ In this document, we propose a uniform terminology for ASMD phenotypes so that the spectrum of disease can be better described and to differentiate ASMD from the eponymous NPD C.

- Infantile neurovisceral ASMD: Also known as NPD A, this is the most severe form of ASMD. Patients have little to no residual ASM activity. Symptom onset occurs in early infancy with rapidly progressive systemic manifestations, including hepatosplenomegaly and pulmonary involvement, neurodegeneration, hypotonia, and failure to thrive. ${ }^{5}$ Psychomotor development may progress normally for several months after birth before plateauing between 6 and 15 months of age and then regressing. Death is typically before 3 years of age and often results from respiratory failure following infection. ${ }^{5}$

- Chronic neurovisceral ASMD: Also known as the intermediate form, NPD A/B or NPD B variant ${ }^{6,7}$ is characterized by slower progression of neurological symptoms and prolonged survival compared to infantile neurovisceral ASMD. Ataxia, gross motor delays, and learning disabilities are commonly seen. Progressive multisystem disease manifestations are similar to or more severe than those observed in chronic visceral ASMD.

- Chronic visceral ASMD: Also known as NPD B, it is characterized by a variable age of onset ranging from infancy to adulthood and slowly progressive multisystem disease manifestations without neurodegeneration. ${ }^{8-11}$ Patients may have a normal life span or die prematurely from disease complications that include respiratory failure, liver failure, and/or hemorrhage. ${ }^{8,12}$ The most common initial presentation is hepatosplenomegaly in early childhood, although mild disease may not be diagnosed until adulthood. It is not uncommon for the splenic volume to be significant (e.g., greater than ten multiples of normal). Common clinical features in childhood include delayed growth and puberty, fatigue, and bone and joint pain. ${ }^{13}$ Other abnormalities include osteopenia and decreased platelet and white blood cell counts. Pulmonary function may worsen over time, and interstitial lung disease and pulmonary infections are common. ${ }^{11,14}$ A proatherogenic lipid profile (mixed dyslipidemia) is seen early in the disease course and some patients develop coronary artery disease. ${ }^{15-17}$ Development of hepatic fibrosis ranging from minimal to cirrhosis is common, ${ }^{18}$ and progression of liver disease contributes to early mortality in some patients. ${ }^{8,19}$ Progressive splenomegaly may result from deposition of sphingomyelin and progressive portal hypertension.

\section{DIFFERENTIAL DIAGNOSIS}

A common clinical presentation for patients with any form of ASMD is hepatosplenomegaly. The liver is the primary affected organ in the chronic visceral form of ASMD, with fibrosis and development of cirrhosis being major morbidities. Transaminases are often increased early in the disease course; therefore, the first referral for ASMD patients may be to a hepatologist or gastroenterologist. Differential diagnoses include malignancy, primary hepatic disease (e.g., fatty liver, autoimmune, chronic hepatitis B-related, cryptogenic cirrhosis), and other LSDs associated with distinct hepatosplenomegaly such as Gaucher disease, NPD C, and lysosomal acid lipase deficiency (LALD). With GM1 and GM2 gangliosidosis, Farber disease, the mucopolysaccharidoses, and other metabolic diseases (e.g., lysinuric protein intolerance, mevalonic aciduria, transaldolase deficiency), hepatosplenomegaly is typically absent or milder than with ASMD; however, exceptions can be expected. Focal liver abnormalities are common in ASMD. Sudden or progressive increases in splenomegaly may result from infection or onset of portal hypertension.

Mixed dyslipidemia is also common for patients with ASMD and may be detected by routine testing ordered by the primarycare physician as part of health maintenance. Lipid profiles are 
Table 1 Clinical features and natural history of ASMD by phenotype

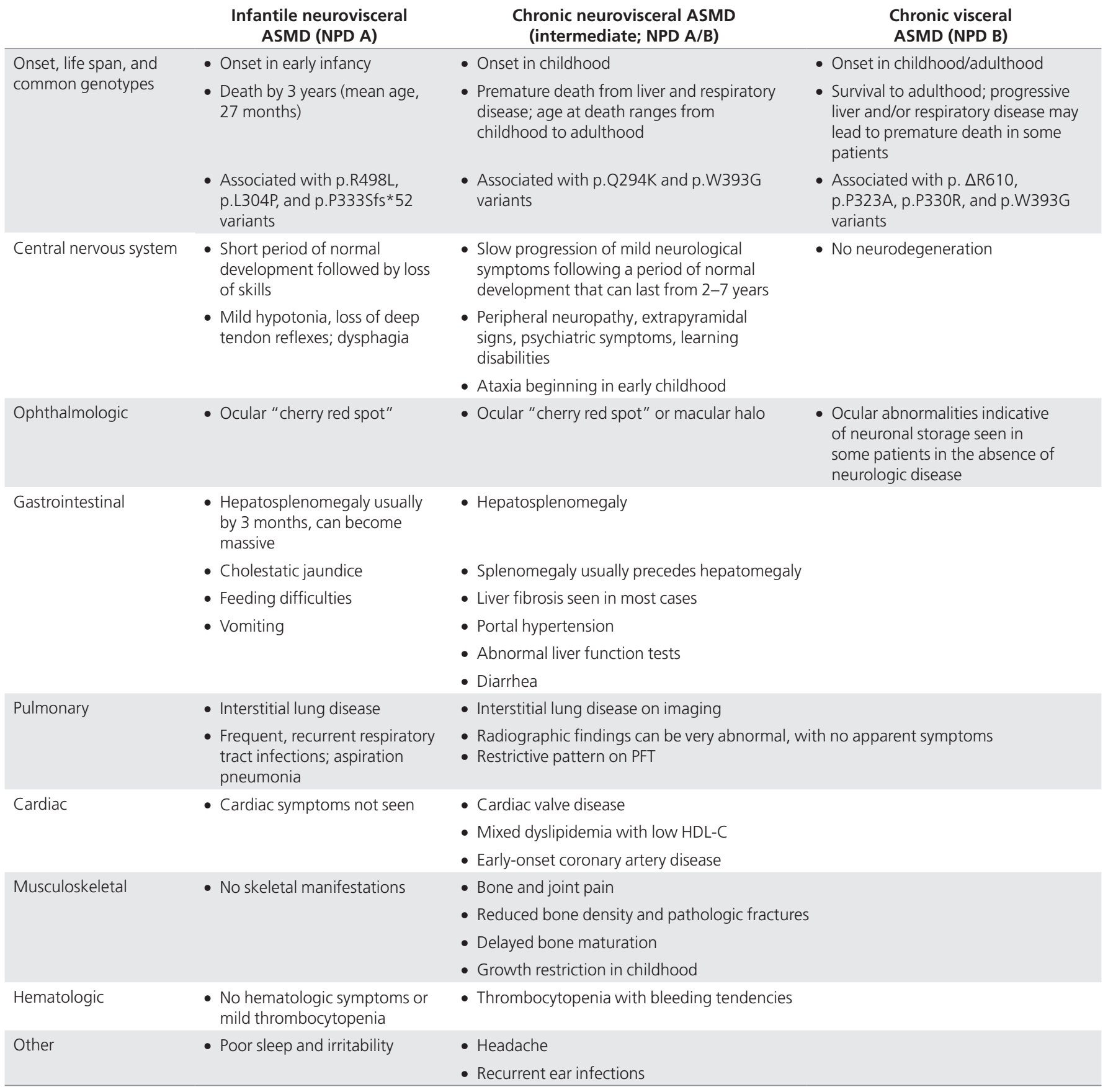

ASMD, acid sphingomyelinase deficiency; NPD, Niemann-Pick disease.

typically characterized by increased LDL cholesterol, VLDL cholesterol, and triglyceride levels, whereas HDL cholesterol levels are substantially decreased., ${ }^{3,15,17}$ A similar lipid profile is seen in LALD, and decreased HDL cholesterol also occurs in patients with Gaucher disease, although the decreases in ASMD are much more severe.

Although some ASMD patients do not have overt respiratory symptoms, most patients have abnormal pulmonary function, which shows a restrictive pattern with low diffusing capacity, and abnormal lung imaging with evidence of interstitial lung disease (ILD). ${ }^{14}$ Adult patients with ASMD may present with lung-only involvement. ${ }^{20}$ This expands the differential diagnosis to include drug and environmental exposures, certain infections, autoimmune disorders, chronic respiratory diseases such as cystic fibrosis, and infectious etiologies such as tuberculosis. Pulmonary disease in ASMD is represented by a range of progressive pathophysiological changes that result in lung volume restriction and impaired gas exchange, including lipid 
accumulation in macrophages within the alveolar septa, bronchial walls, and pleura. ${ }^{8}$ An additional factor may be an inflammatory component observed in the ASM knockout mouse model that drives the recruitment of macrophages to the lung. ${ }^{21}$

\section{DIAGNOSTIC LABORATORY TESTING}

It is the consensus of the advisory panel that when there is suspicion of ASMD, an enzyme assay for ASM activity should always be performed first, with gene sequencing to follow once the biochemical diagnosis has been confirmed. Simultaneous determination of glucocerebrosidase activity to distinguish ASMD from Gaucher disease is recommended. Gene sequencing can be diagnostic if two pathogenic mutations are detected; however, most genetic variants are not pathogenic. Therefore, gene sequencing should not be the first approach to diagnosis because it typically takes longer than enzyme activity and may delay definitive diagnosis in patients affected with a disorder other than ASMD. Demonstration of missing or significantly diminished enzyme activity remains the proof of the presence of the disease, especially in view of the many unique mutations or genetic variants of unknown significance. Results from other clinical and laboratory assessments, such as the presence of characteristic lipid-laden foam cells present in the liver, spleen, airways, and bone marrow, together with low platelet levels and mixed dyslipidemia (low HDL cholesterol with high levels of LDL cholesterol and triglycerides), although highly suggestive of ASMD, are not substitutes for the need to obtain confirmatory enzyme test results.

To standardize testing and ensure its diagnostic value, it is suggested that laboratory centers of excellence should be established based on the minimum number of tests per month, ISO 15189 or similar accreditation, and access to physicians involved in patient care. At minimum, preferred methodologies should be used by all testing laboratories as described here. Of note, residual enzyme activity should not be used as a predictor of phenotype; instead, positive enzyme testing should be used in concert with molecular and clinical assessments.

\section{Best practice for enzyme testing}

Tandem mass spectrometry (MS/MS) is considered the method of choice for assaying ASM activity rather than fluorometric assays due to enhanced analytical range and more accurate assessment of enzyme activity in the lower ranges of detection, and rather than radiolabeled native sphingomyelin substrate due to the need for licensing for use of radiochemicals. Comparisons with fluorometry have determined that there is better differentiation between unaffected and affected individuals with MS/MS assays. ${ }^{22}$ Fluorometric substrates for ASMD are known to result in high false-negative rates for individuals who have the p.Q294K (Q292K nomenclature used in reference) mutation, which yields anomalous high enzyme activity readings. ${ }^{23}$ In addition to better substrate specificity and no need for radiochemicals, tandem mass spectrometry allows for multiplexing of the measurement of enzyme activities that are included in the differential diagnosis of ASMD, thereby expediting diagnosis and reducing cost. Currently, this includes Gaucher disease (glucocerebrosidase activity). In contrast, fluorometry using DBS for glucocerebrosidase assessment yields unreliable results and should not be used. In the future, acid lipase (acid lipase deficiency) should be added to the MS/ MS panel as well because the signs and symptoms may mimic ASMD and ERT is now available for this disorder.

ASM enzyme activity may be measured using isolated peripheral blood leukocytes, fibroblasts, and DBS. Laboratories that accept samples from around the world usually require whole blood, which permits use of the same sample for second-tier testing. DBS that are very stable may be used when it is difficult to ship liquid blood samples to reference laboratories. ${ }^{24}$ However, limitations to DBS testing include the potential effects of anemia and recent transfusions on results. Therefore, it is important for transfusions to be stated on the request form, including the time elapsed since the last transfusion and transfusion type, because plasma transfusion may have more of an impact on enzyme tests than erythrocyte transfusions. Cultured skin fibroblasts may be preferred for confirmation of enzyme activity in equivocal cases. As noted, because ASMD and Gaucher disease have several clinical manifestations in common, simultaneous enzyme testing for ASM and glucocerebrosidase should be obtained either as a multiplex test using tandem MS analysis or in parallel with singleplex testing.

\section{Gene sequencing}

The ASM gene (SMPD1) has been cloned and mapped to chromosome 11p15.1-15.4, and more than 180 mutations have been described. ${ }^{4,25}$ There are currently two numbering systems for SMPD1 mutations as a result of two reference sequences (GenBank Accession Numbers NM_000543.4 and M81780.1) that differ by length polymorphism within the signal peptide region. References to mutations should include the appropriate reference sequence for clarity; in this article, the NM_000543.4 sequence was used. Gene sequencing is recommended after confirmation of the diagnosis by demonstration of reduced ASM activity.

There is a great deal of heterogeneity among SMPD1 mutations, and most mutations are "private," occurring in only one or a few families. However, predictions regarding genotype/ phenotype correlations are possible in some cases for which "common" mutations have been found. Frameshift mutations due to splicing, small and large insertions and deletions, and splicing defects typically result in little or no residual ASM activity and are more likely to contribute to severe ASMD. Missense and certain other lesions (such as in-frame codon deletions) that retain significant residual activity (e.g., $>5 \%$ of wild-type ASM activity depending on the cell and assay system) are more likely to result in chronic ASMD. Inheritance is autosomal recessive; therefore, the presence of two loss-of-function alleles is required to develop ASMD. Phenotype will depend on the combination of these alleles.

As noted, in some cases "common" SMPD1 mutations have been found in multiple unrelated families. These common 
mutations provide the most reliable genotype/phenotype correlations. For example, three mutations account for more than $90 \%$ of infantile neurovisceral ASMD in the Ashkenazi Jewish population: p.R498L, p.L304P, and p.P333Sfs. ${ }^{26}$ The most common mutation in patients with the chronic visceral form of disease is p. $\Delta$ R610, ${ }^{27}$ which is found in $15-20 \%$ of all North American and Western European patients and is also prevalent in patients from North Africa. p. $\Delta$ R610 is associated with a higher amount of residual enzyme activity and is considered neuroprotective when either homoallelic or heteroallelic. A 10-year longitudinal study of 29 patients with the chronic visceral form of ASMD showed that attenuated disease is associated with homozygosity for mutations such as p. $\Delta \mathrm{R} 610$, p.P325A, and p.P332R. ${ }^{10}$ Common mutations detected in the chronic neurovisceral form include p.Q294K ${ }^{6,7}$ and p.W393G, ${ }^{28}$ although the p.W393G mutation is also observed in patients with chronic visceral ASMD. There is ongoing research regarding identification and characterization of novel SMPD1 mutations and their phenotypic correlations, ${ }^{25,29}$ which are likely to be improved by development of better computer modeling based on ASM structure.

\section{Biomarkers for ASMD}

Biomarker assays may be useful for disease monitoring once the diagnosis of ASMD is made. However, this is an area of ongoing research, and the finding of increased levels of one or more markers is never sufficient to diagnose ASMD, although they may be helpful for assessing disease severity. The role of ASMD biomarkers may become of greater importance to monitor treatment responses as ERT becomes available. The following is a brief summary of several biomarkers that have been studied regarding their utility as indicators for ASMD.

Plasma chitotriosidase, which is a biomarker of macrophage activation, is markedly increase in several LSDs, including Gaucher disease ${ }^{30}$ and chronic ASMD. ${ }^{31}$ In Gaucher disease, it has been proven to be useful for monitoring disease severity and effectiveness of ERT. ${ }^{32}$ Although levels observed in ASMD are not as high as those measured in patients with Gaucher disease, chitotriosidase may serve as a useful biomarker for chronic ASMD. ${ }^{33,34}$ However, up to $6 \%$ of individuals have a recessively inherited deficiency in chitotriosidase, ${ }^{35}$ and polymorphisms in the chitotriosidase gene that compromise assay results ${ }^{36}$ can result in incorrect interpretations of chitotriosidase levels measured in plasma. Plasma CCL18 levels are also increased in Gaucher disease, ${ }^{37}$ where it can serve as a surrogate marker for disease activity, and in patients with ASMD. ${ }^{34}$ This marker is of particular use when patients have chitotriosidase deficiency.

Plasma lysosphingolipids have been shown to be biomarkers of sphingolipidosis in several LSDs. For example, glucosylsphingosine is a specific and sensitive biomarker for Gaucher disease and is not increased in other LSDs. ${ }^{38}$ Lysosphingomyelin is increased in DBS and plasma from patients with chronic ASMD, suggesting that it may have use as a biomarker; however, additional studies are needed. ${ }^{39}$
As noted, dyslipidemia is a common feature of patients with ASMD, even at a very early age. ${ }^{3,15,17}$ Oxysterols are oxygenated derivatives of cholesterol that are typically rapidly removed from the body during normal cholesterol metabolism but that have been shown to be increased in NPD C, chronic ASMD, and LALD and may correlate with hepatic disease in patients with NPD C. ${ }^{40}$

\section{DIAGNOSTIC ALGORITHM}

Based on the common presenting and associated symptoms of ASMD, differential diagnoses, and approaches to diagnostic testing described in the previous sections, an algorithm for the diagnosis of the infantile neurovisceral form of ASMD and the chronic neurovisceral and visceral forms of ASMD presenting in childhood is presented in Figure 1. An algorithm for diagnosis of ASMD presenting after childhood is shown in Figure 2.

Following biochemical and molecular analyses to confirm the diagnosis of ASMD, predictions of phenotypic outcomes will permit more appropriate patient care and family counseling. Physicians will rely on clinical assessments to predict the phenotype and clinical course for pediatric patients when SMPD1 mutations of unknown pathogenicity are identified.

\section{CONCLUSIONS}

Patients with ASMD present with a large phenotypic spectrum of nonspecific disease manifestations that can lead to considerable diagnostic delay and missed cases. Early diagnosis of ASMD is a priority for appropriate management.

\section{APPENDIX: CASE VIGNETTES}

The following are fictitious case vignettes based on real-life cases and are meant to describe the symptoms that may help differentiate between the different forms of ASMD as well as highlight the difficulty in establishing a proper clinical diagnosis based solely on a patient's genotype.

\section{Case 1: Infantile neurovisceral ASMD}

A male infant with Eastern European Jewish ancestry presented at 3 months of age with hepatosplenomegaly. At 4 months of age, notable signs and symptoms included elevated liver transaminases, gastroesophageal reflux, and failure to thrive. In addition, at 4 months of age, the patient was hospitalized for failure to thrive. Hypotonia with poor head control was noted. Enzyme assay for ASM activity was performed at 5 months of age and the diagnosis of ASMD was made. Genotyping showed a common mutation associated with infantile neurovisceral ASMD in patients of Eastern European Jewish ancestry as well as a novel frameshift mutation. At 10 months of age, the patient was unable to sit independently or hold a bottle. The patient had progressive failure to thrive, liver dysfunction, and neurologic deterioration. He died at 2 years of age.

Comment: This is the classic presentation and course for infantile neurovisceral ASMD. The severity of the symptoms 


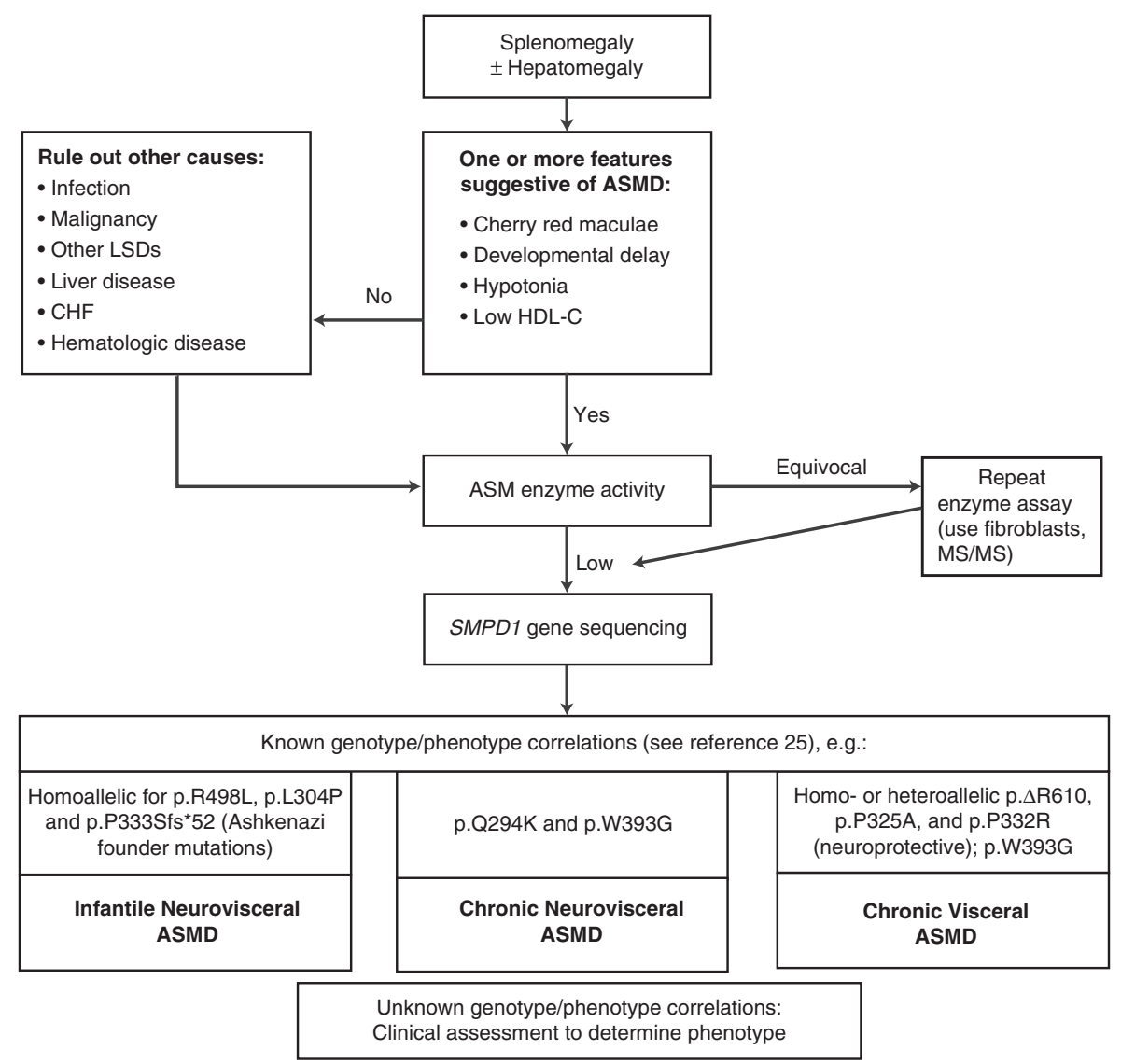

Abbreviations: $\mathrm{ASM}$ = acid sphingomyelinase; $\mathrm{ASMD}=$ acid sphingomyelinase deficiency; $\mathrm{CHF}$ = congestive heart failure; HDL-C = high density lipoprotein cholesterol; LSD = lysosomal storage disorder; $M S / M S=$ tandem mass spectrometry; SMPD1 = ASM gene

Figure 1 Diagnostic algorithm for acid sphingomyelinase deficiency presenting in infancy and childhood.

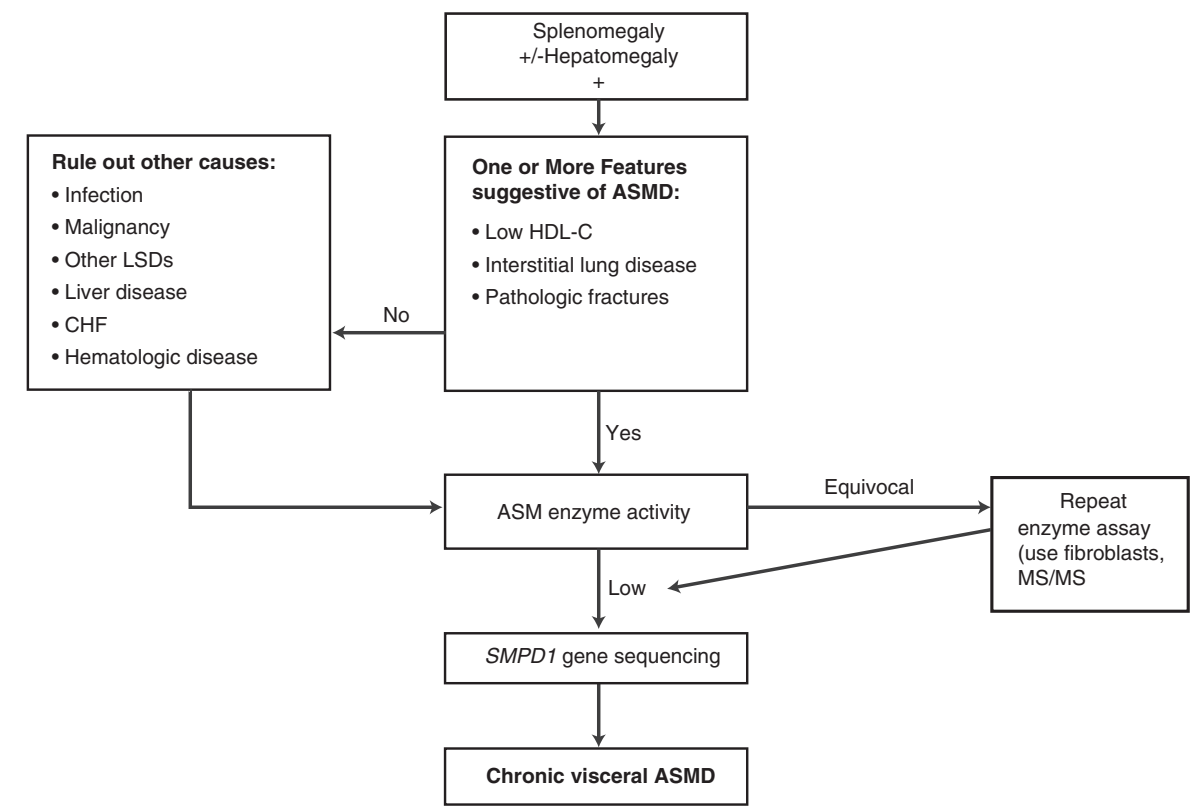

Abbreviations: $A S M=$ acid sphingomyelinase $A S M D=$ acid sphingomyelinase deficiency; $\mathrm{CHF}=$ congestive heart failure; $\mathrm{HDL}-\mathrm{C}=$ high density lipoprotein cholesterol; $\mathrm{LSD}=$ lysosomal storage disorder; $\mathrm{MS} / \mathrm{MS}=$ tandem mass spectrometry; SMPD1 = ASM gene

Figure 2 Diagnostic algorithm for acid sphingomyelinase deficiency presenting after childhood. 
at a very young age together with the relevant ancestry should prompt early medical attention and diagnosis.

\section{Case 2: Chronic neurovisceral ASMD}

A male child presented with marked abdominal distension, chronic diarrhea, and failure to thrive at 6 months of age and was referred to a cystic fibrosis specialist. Other signs and symptoms of note included severe hepatosplenomegaly and hypotrophic muscles. Suspicion of an LSD resulted in referral to a metabolic specialist, who diagnosed ASMD with an enzyme assay. The patient was able to sit without support at 10 months of age. Laboratory findings included dyslipidemia, elevated liver transaminases, thrombocytopenia, and leukopenia. Lung involvement included interstitial pneumopathy with ground-glass aspect. Genotyping revealed one missense allele associated with infantile neurovisceral ASMD or with chronic neurovisceral ASMD and one nonsense allele associated with infantile neurovisceral ASMD. By 6 years of age, the patient also presented with developmental delay and recurrent respiratory infections.

Comment: Although some of the presenting features of this patient are similar to those of patients with infantile neurovisceral ASMD, the child's developmental milestones did not regress as in patients with infantile neurovisceral ASMD. The diagnosis at 9 months of age accompanied by a genotype that is insufficient to distinguish between infantile neurovisceral and chronic neurovisceral disease should make the monitoring of clinical signs important for the prediction of the clinical course.

\section{Case 3: Pediatric-onset chronic visceral ASMD}

A female child had normal growth and development for the first 2 years of life but was then noted to have splenomegaly at a routine pediatric visit. The child underwent a diagnostic workup for pediatric causes of splenomegaly including $\mathrm{CBC}$, viral studies, and liver function tests that were all normal. The patient was eventually diagnosed with ASMD at age 6 when she developed severe nosebleeds and underwent a hematologic evaluation for thrombocytopenia, at which time she was noted to have hepatosplenomegaly and the possibility of a storage disease was added to the differential diagnosis. Genotyping revealed two missense mutations. The patient is now 10 years old and at the age-appropriate grade level in school, but she has not achieved appropriate height and suffers from fatigue. She has persistently increased transaminases and dyslipidemia with decreased HDL levels and increased triglycerides. Chest X-ray showed increased interstitial markings.

Comment: The patient presented with organomegaly, which is a common presenting sign in ASMD. The delay in diagnosis of 4 years is very common with patients with chronic visceral ASMD because they frequently have a lengthy diagnostic journey.

\section{Case 4: Adult-onset chronic visceral ASMD}

A middle-aged male patient was diagnosed with hepatosplenomegaly following an ultrasound evaluation for abdominal pain.
A bone marrow biopsy showed Niemann-Pick cells. ASM activity was within the affected range, and molecular sequencing of the SMPD1 gene revealed compound heterozygosity for $\triangle \mathrm{R} 610$ and a mutation associated with infantile neurovisceral ASMD. Hepatosplenomegaly was present on abdominal MRI, as was an incidental finding of a small adrenal mass thought to be consistent with sphingomyelin deposition. Prior to the diagnosis, the patient considered himself to be in good health, although he had occasional shortness of breath with activity and was being treated for hypercholesterolemia. After ASMD was confirmed, chest X-ray and chest CT revealed interstitial lung disease predominantly within the lung bases. Pulmonary function testing revealed a restrictive pattern with low diffusing capacity and HDL cholesterol was low; platelets and liver function test results were within the normal range.

Comment: This patient had hepatosplenomegaly, an abnormal lipid profile with low HDL, and lung findings that were consistent with chronic visceral ASMD, although he was not diagnosed until much later in life. This case demonstrates the milder end of the phenotypic spectrum of ASMD and the features (organomegaly, respiratory issues, and mixed lipidemia) that are indicative of ASMD onset after childhood.

\section{ACKNOWLEDGMENTS}

The results of this paper were presented in part at the SSIEM 2016 Annual Symposium (Poster 465), and at the 2017 World Symposium (Poster 357).

\section{DISCLAIMER}

This diagnostic guideline is intended as an educational resource and represents the opinions of the authors, and is not representative of recommendations or policy of the American College of Medical Genetics and Genomics (ACMG). The information should be considered a consensus based on expert opinion, as more comprehensive levels of evidence were not available in the literature in all cases.

\section{DISCLOSURE}

C.D-V. has received research grants, investigator fees, speaker honoraria, and reimbursement of travel expenses to attend scientific meetings from Sanofi Genzyme. E.H.S. is an inventor of several patents that have been licensed by Mount Sinai to Sanofi Genzyme for the treatment and diagnosis of ASMD. He is also a consultant for Sanofi Genzyme and receives a research grant for the study of ASMD. K.E.M. has received investigator fees, speaker honoraria, and reimbursement of travel expenses from Sanofi Genzyme to attend scientific meetings. M.P.W. has received travel grants, honoraria, and investigator fees from Sanofi Genzyme. O.L. has received travel grants and speaker honoraria from Sanofi Genzyme. P.K.M. has received research grants and lecture honoraria from Sanofi Genzyme. R.G. has received investigator fees, speaker honoraria, and reimbursement of travel expenses from Sanofi Genzyme to attend scientific meetings. Z.L. has received travel grants, honoraria, and research grants from Sanofi Genzyme. P.H. and M.M.M. have no conflicts of interest. 
Sanofi Genzyme provided support, including an honorarium for the authors to meet and develop the consensus document. The opinions represent those of the authors based on their clinical expertise and experience and do not reflect those of Sanofi Genzyme. Manuscript preparation and editing were provided by Patrice C. Ferriola and were funded by Sanofi Genzyme

\section{REFERENCES}

1. Schuchman EH, Desnick RJ. Niemann-Pick disease types A and B: acid sphingomyelinase deficiencies. In: Valle D, Beaudet A, Vogelstein B, et al. (eds). OMMBID - The Online Metabolic and Molecular Bases of Inherited Disease. McGraw Hill: New York, 2013. http://ommbid.mhmedical.com/content.aspx? bookid=971\&sectionid=62643414. Accessed January 2015.

2. Kingma SD, Bodamer OA, Wijburg FA. Epidemiology and diagnosis of lysosomal storage disorders; challenges of screening. Best Pract Res Clin Endocrino/ Metab 2015;29:145-157.

3. Wasserstein MP, Jones SA, Soran $\mathrm{H}$, et al. Successful within-patient dose escalation of olipudase alfa in acid sphingomyelinase deficiency. Mol Genet Metab 2015;116:88-97.

4. Schuchman EH, Wasserstein MP. Types A and B Niemann-Pick disease. Best Pract Res Clin Endocrino/ Metab 2015:29:237-247.

5. McGovern MM, Aron A, Brodie SE, Desnick RJ, Wasserstein MP. Natural history of Type A Niemann-Pick disease: possible endpoints for therapeutic trials. Neurology 2006;66:228-232.

6. Pavlů-Pereira $\mathrm{H}$, Asfaw B, Poupctová $\mathrm{H}$, et al. Acid sphingomyelinase deficiency. Phenotype variability with prevalence of intermediate phenotype in a series of twenty-five Czech and Slovak patients. A multi-approach study. J Inherit Metab Dis 2005;28:203-227.

7. Wasserstein MP, Aron A, Brodie SE, Simonaro C, Desnick RJ, McGovern MM Acid sphingomyelinase deficiency: prevalence and characterization of an intermediate phenotype of Niemann-Pick disease. J Pediatr 2006;149:554-559.

8. McGovern MM, Lippa N, Bagiella E, Schuchman EH, Desnick RJ, Wasserstein MP. Morbidity and mortality in type B Niemann-Pick disease. Genet Med 2013;15:618-623.

9. McGovern MM, Wasserstein MP, Giugliani R, et al. A prospective, crosssectional survey study of the natural history of Niemann-Pick disease type B. Pediatrics 2008;122:e341-e349.

10. Wasserstein MP, Desnick RJ, Schuchman EH, et al. The natural history of type B Niemann-Pick disease: results from a 10-year longitudinal study. Pediatrics 2004; 114:e672-e677

11. Hollak CE, de Sonnaville ES, Cassiman D, et al. Acid sphingomyelinase (Asm) deficiency patients in The Netherlands and Belgium: disease spectrum and natural course in attenuated patients. Mol Genet Metab 2012;107: 526-533.

12. Cassiman D, Packman S, Bembi B, et al. Cause of death in patients with chronic visceral and chronic neurovisceral acid sphingomyelinase deficiency (NiemannPick disease type $B$ and $B$ variant): Literature review and report of new cases. Mol Genet Metab 2016;118:206-213.

13. Wasserstein M, Godbold J, McGovern MM. Skeletal manifestations in pediatric and adult patients with Niemann Pick disease type B. J Inherit Metab Dis 2013;36:123-127.

14. Mendelson DS, Wasserstein MP, Desnick RJ, et al. Type B Niemann-Pick disease: findings at chest radiography, thin-section $\mathrm{CT}$, and pulmonary function testing. Radiology 2006;238:339-345

15. McGovern MM, Pohl-Worgall T, Deckelbaum RJ, et al. Lipid abnormalities in children with types A and B Niemann Pick disease. J Pediatr 2004;145:77-81.

16. Orchard EA, Kempson D, Lindsay DC. Niemann-Pick disease type B with severe coronary artery disease and early recurrence after coronary artery bypass grafting. J Clin Lipido/ 2009;3:57-58.

17. Ishii H, Takahashi T, Toyono M, et al. Acid sphingomyelinase deficiency: cardiac dysfunction and characteristic findings of the coronary arteries. J Inherit Metab Dis 2006;29:232-234

18. Thurberg BL, Wasserstein MP, Schiano T, et al. Liver and skin histopathology in adults with acid sphingomyelinase deficiency (Niemann-Pick disease type B). Am J Surg Pathol 2012;36:1234-1246.

19. Labrune $P$, Bedossa $P$, Huguet $P$, Roset $F$, Vanier MT, Odievre M. Fatal liver failure in two children with Niemann-Pick disease type B. J Pediatr Gastroentero/ Nutr 1991;13:104-109.

20. Nicholson AG, Florio R, Hansell DM, et al. Pulmonary involvement by NiemannPick disease. A report of six cases. Histopathology 2006;48:596-603.
21. Schuchman EH. The pathogenesis and treatment of acid sphingomyelinasedeficient Niemann-Pick disease. J Inherit Metab Dis 2007;30:654-663.

22. Gelb MH, Scott CR, Turecek F. Newborn screening for lysosomal storage diseases. Clin Chem 2015;61:335-346.

23. Harzer K, Rolfs A, Bauer P, et al. Niemann-Pick disease type A and B are clinically but also enzymatically heterogeneous: pitfall in the laboratory diagnosis of sphingomyelinase deficiency associated with the mutation Q292 K. Neuropediatrics 2003:34:301-306

24. Civallero G, Michelin K, de Mari J, et al. Twelve different enzyme assays on dried-blood filter paper samples for detection of patients with selected inherited lysosomal storage diseases. Clin Chim Acta 2006;372:98-102.

25. Zampieri S, Filocamo M, Pianta A, et al. SMPD1 mutation update: database and comprehensive analysis of published and novel variants. Hum Mutat 2016;37:139-147.

26. Wasserstein M, Schuchman E. Acid sphingomyelinase deficiency. In: Pagon R, Adamm M, Ardinger $\mathrm{H}$, et al. (eds). GeneReviews. University of Washington: Seattle, WA, 18 June 2015.

27. Simonaro CM, Desnick RJ, McGovern MM, Wasserstein MP, Schuchman EH. The demographics and distribution of type B Niemann-Pick disease: novel mutations lead to new genotype/phenotype correlations. Am J Hum Genet 2002;71:1413-1419.

28. Mihaylova V, Hantke J, Sinigerska I, et al. Highly variable neural involvement in sphingomyelinase-deficient Niemann-Pick disease caused by an ancestral Gypsy mutation. Brain 2007;130(Pt 4):1050-1061.

29. Desnick JP, Kim J, He X, Wasserstein MP, Simonaro CM, Schuchman EH. Identification and characterization of eight novel SMPD1 mutations causing types A and B Niemann-Pick disease. Mol Med 2010;16:316-321.

30. Hollak CE, van Weely S, van Oers MH, Aerts JM. Marked elevation of plasma chitotriosidase activity. A novel hallmark of Gaucher disease. J Clin Invest 1994:93:1288-1292

31. Sheth JJ, Sheth FJ, Oza NJ, Gambhir PS, Dave UP, Shah RC. Plasma chitotriosidase activity in children with lysosomal storage disorders. Indian J Pediatr 2010;77:203-205.

32. van Dussen L, Hendriks EJ, Groener JE, Boot RG, Hollak CE, Aerts JM. Value of plasma chitotriosidase to assess non-neuronopathic Gaucher disease severity and progression in the era of enzyme replacement therapy. J Inherit Metab Dis 2014;37:991-1001.

33. Kadali S, Kolusu A, Sunkara S, Gummadi MR, Undamatla J. Clinical evaluation of chitotriosidase enzyme activity in Gaucher and Niemann Pick A/B diseases: A retrospective study from India. Clin Chim Acta 2016;457:8-11.

34. Brinkman J, Wijburg FA, Hollak CE, et al. Plasma chitotriosidase and CCL18: early biochemical surrogate markers in type B Niemann-Pick disease. J Inherit Metab Dis 2005;28:13-20.

35. Boot RG, Renkema GH, Verhoek $M$, et al. The human chitotriosidase gene. Nature of inherited enzyme deficiency. J Biol Chem 1998;273:25680-25685.

36. Bussink AP, Verhoek M, Vreede J, et al. Common G102S polymorphism in chitotriosidase differentially affects activity towards 4-methylumbelliferyl substrates. FEBS J 2009;276:5678-5688.

37. Deegan PB, Moran MT, McFarlane I, et al. Clinical evaluation of chemokine and enzymatic biomarkers of Gaucher disease. Blood Cells Mol Dis 2005;35:259-267.

38. Murugesan V, Chuang WL, Liu J, et al. Glucosylsphingosine is a key biomarker of Gaucher disease. Am J Hematol 2016;91:1082-1089.

39. Chuang WL, Pacheco J, Cooper S, et al. Lyso-sphingomyelin is elevated in dried blood spots of Niemann-Pick B patients. Mol Genet Metab 2014;111:209-211.

40. Boenzi S, Deodato F, Taurisano R, Goffredo BM, Rizzo C, Dionisi-Vici C. Evaluation of plasma cholestane-3 $3,5 \alpha, 6 \beta$-triol and 7-ketocholesterol in inherited disorders related to cholesterol metabolism. J Lipid Res 2016;57:361-367.

(c) (1) $\Theta$ This work is licensed under a Creative Commons Attribution-NonCommercial-NoDerivs 4.0 International License. The images or other third party material in this article are included in the article's Creative Commons license, unless indicated otherwise in the credit line; if the material is not included under the Creative Commons license, users will need to obtain permission from the license holder to reproduce the material. To view a copy of this license, visit http://creativecommons.org/licenses/by-nc-nd/4.0/

(c) The Author(s) (2017) 